\title{
Zinkmangeldermatitis
}

\section{Zinc Deficiency Disorder}

\author{
Autoren \\ I. Feldmann-Böddeker, T. Christoph \\ Institut \\ Hautklinik (Chefärztin: Dr. med. I. Feldmann-Böddeker), DRK-Krankenhaus Chemnitz-Rabenstein
}

\section{Bibliografie}

DOI $10.1055 / \mathrm{s}-2008-1077702$

Online-Publikation: 27.11.2008

Akt Dermatol 2009; 35:

43-45 @ Georg Thieme

Verlag KG Stuttgart · New York

ISSN 0340-2541

Korrespondenzadresse

\section{Dr. med. Ingrid Feldmann-} Böddeker

Hautklinik, DRK-Krankenhaus

Chemnitz-Rabenstein

Unritzstr. 23

09117 Chemnitz

feldmann-boeddeker.ingrid@

drk-chemnitz.de

\section{Zusammenfassung \\ $\nabla$}

Bei der Symptomenkombination akral betonte Hautveränderungen, diffuse Alopezie und Diarrhoe sollte differenzialdiagnostisch auch an eine Zinkmangeldermatitis gedacht werden. Neben

\section{Einleitung \\ $\nabla$}

Zink ist ein essenzielles Spurenelement, das regelmäßig exogen mit der Nahrung zugeführt werden muss. Nach Angaben der deutschen Gesellschaft für Ernährung findet sich bei $75 \%$ aller Bundesbürger eine mangelhafte Zinkzufuhr. Abgegrenzt werden muss von der erworbenen Zinkmangeldermatitis, die hereditäre Acrodermatitis enteropathica.

\section{Anamnese \\ $\nabla$}

Eine 38-jährige Patientin wurde wegen therapieresistenter generalisierter juckender Hautveränderungen zur stationären Diagnostik und Therapie in unsere Hautklinik eingewiesen. Erytheme waren erstmalig 3 Monate zuvor an den Füßen und Unterschenkeln aufgetreten. Trotz ambulanter Anwendung diverser topischer Glukokortikoide nahmen die Effloreszenzen an Intensität zu und breiteten sich schließlich auf das gesamte Integument aus. Die Patientin klagte über massiven Juckreiz. In der weiteren Anamnese war lediglich eine Katarakt bekannt.

\section{Hautbefund bei Aufnahme \\ $\nabla$}

Unter Aussparung der großen Gelenkbeugen waren am gesamten Integument scharf begrenzte, großflächig konfluierende, schuppende, teils keratotisch belegte Erytheme mit dazwischen lie- einem erniedrigten Zinkspiegel im Serum kann auch eine erniedrigte alkalische Phosphatase hinweisend auf diese Erkrankung sein. Bei der erworbenen Zinkmangeldermatitis muss eine eingehende Diagnostik zur Abklärung der Ursache des Zinkmangels angeschlossen werden.

genden erosiv-nässenden Erythemen sichtbar, weiterhin disseminierte Exkoriationen. Besonders ausgeprägt war der Hautbefund an den Akren ( $\bullet$ Abb. 1). Neben einer Perlèche fielen auch perioral schuppende Erytheme auf. Auffällig waren weiterhin eine ausgeprägte Kachexie, eine Xerosis cutis und eine diffuse Alopezie der Kopfhaut. Auf beiden Fußrücken bestanden Schnittverletzungen (anamnestisch waren diese durch eine heruntergefallene Flasche entstanden).

\section{Untersuchungsbefunde \\ $\nabla$}

\section{Laborbefunde}

Pathologisch waren: Zinkim Serum 7,1 $\mu \mathrm{mol} / \mathrm{l}$ (Referenzbereich 10,5-17,5 $\mathrm{mol} / \mathrm{l})$, $\mathrm{Hb} 7,2 \mathrm{mmol} / \mathrm{l}$, Hk 0,34, Erythrozyten 3,52 Tpt/l, MCH 2,04 fmol, Neutr. 0,77, Lym. 0,14, Kalium 3,2 mmol/l, Calcium $2,09 \mathrm{mmol} / \mathrm{l}$, ASAT $0,96 \mu \mathrm{mol} / \mathrm{sl}, \quad$ Gamma-GT $9,6 \mu \mathrm{mol} / \mathrm{sl}$, AP $6,6 \mu \mathrm{mol} / \mathrm{sl}$, Amylase 0,08 $\mu \mathrm{mol} / \mathrm{sl}$, CRP60 mg/l.

Urinstatus: Protein $300 \mathrm{mg} / \mathrm{l}$, Leukozyten $70 \mathrm{c} / \mu \mathrm{l}$, Erythrozyten $10 \mathrm{c} / \mu \mathrm{l}$, mäßig Bilirubin.

Unauffällig waren: übrige Blutbildparameter, Gerinnungsstatus, Natrium, ALAT, LDH, Kreatinin, Blutzucker, Cholesterin und BSG sowie Stuhl auf Blut.

Atopiediagnostik: Gesamt-IgE 208 kU/l, Erlanger Atopiekriterien: 6 Punke - atopische Hautdiathese unwahrscheinlich.

EKG: Sinustachykardie, Herzfrequenz 100/', sonst unauffällig. 
Röntgen-Thorax in 2 Ebenen: Unauffälliger Befund. Oberbauch-Sonografie und ableitende Harnwege: Leberzirrhose mit minimalem Aszitessaum perihepatisch.

\section{Therapie und Verlauf \\ $\nabla$}

Aufgrund des vorliegenden Hautbefundes und des erniedrigten Zinkserumspiegels wurde die Diagnose einer erworbenen Zinkmangeldermatitis gestellt. Unter einer Zinksubstitution mit Unizink 50 Tbl. 1 pro Tag (Zink DL-aspartat) und einer Lokaltherapie mit Triclosan $2 \%$-Triamcinolon 0,1\%-DAC-Basiscreme sowie intensiver rückfettender Hautpflege war der Hautbefund innerhalb der dreiwöchigen stationären Therapie langsam, aber sehr gut rückläufig. Zur Linderung des Juckreizes wurden FenistilDragees und zusätzlich Xusal-Tbl. verordnet. Bei Entlassung war der Zinkspiegel auf 9,9 $\mu \mathrm{mol} / \mathrm{l}$ angestiegen. Empfohlen wurde zunächst die weitere Gabe von $50 \mathrm{mg}$ Unizink-Tbl. pro Tag. Nach Anhebung des Zinkspiegels in den Referenzbereich sollte langfristig weiter $20 \mathrm{mg}$ Zink pro Tag verabreicht werden. Ihr Körpergewicht hatte die Patientin trotz regelmäßiger Intervention durch unsere klinischen Psychologen bis zur Entlassung von 38,7 auf $38 \mathrm{~kg}$ weiter reduziert. Zur weiteren Therapie der von den Psychologen diagnostizierten Anorexia nervosa vom bulimischen Typ und des Alkoholabusus wurde die Patientin schließlich in eine psychiatrische Tagesklinik verlegt.

\section{Diskussion \\ $\nabla$}

Als Ursache des erworbenen Zinkmangels wurden bei unserer Patientin eine exzessiv einseitige Ernährung bei Anorexia nervosa vom bulimischen Typ, der Alkoholabusus und die hierdurch bedingte Leberzirrhose diagnostiziert. Während des letzten Jahres wurden in unserer Hautklinik zwei weitere Patienten, die an einer ausgedehnten erworbenen Zinkmangeldermatitis bei Leberzirrhose erkrankt waren, erfolgreich durch Zinksubstitution behandelt.

Zink ist ein essenzielles Spurenelement, das exogen mit der Nahrung zugeführt werden muss. Es ist Bestandteil zahlreicher Enzyme, z. B. der Lactatdehydrogenase und alkalischen Phosphatase. Auch der Keratinisierungsprozess der Haut benötigt Zink. So hat die Epidermis einen hohen Zinkgehalt im Filaggrin der Keratohyalingranula und in der in der Epidermis befindlichen alkalischen Phosphatase. Der tägliche Zinkbedarf eines Erwachsenen beträgt 10 bis $15 \mathrm{mg}$. Das Zinkreservoir des Körpers beträgt 2 bis 3 g. $6 \%$ des Zinkreservoirs liegen in der Haut. Hohe Zinkkonzentrationen finden sich in Augen, Hoden, Leber, Knochen, Pankreas und Haaren. Symptome eines Zinkmangels können bereits nach 1 Monat zinkfreier Ernährung auftreten. Hauptsymptome sind neben einer diffusen Alopezie und Diarrhoe, schuppende und erosiv nässende, gegebenenfalls mit Blasen und Pusteln belegte Erytheme mit einer Betonung der Akralbereiche sowie periorifiziell. Eine bakterielle und mykotische Superinfektion sowie Paronychie werden ebenfalls häufig beobachtet. Unerkannter Zinkmangel bei Kindern führt schließlich auch zu Geruchs- und Geschmackstörungen, Hypogonadismus und körperlicher und geistiger Retardierung. Eine orale oder intravenöse Zinksubstitution von 1 bis $2 \mathrm{mg}$ pro kg Körpergewicht bringt die Hautveränderungen innerhalb weniger Wochen zur Abheilung. Häufig muss eine lebenslange Zinksubstitution angeschlossen werden. Ergänzend sollte eine zinkreiche Ernährung



Abb. 1 Rechtes Knie.

empfohlen werden. Einen hohen Zinkgehalt weisen folgende Nahrungsmittel auf: Austern und Meeresfrüchte, Weizenkeime, Pinienkerne, Linsen, Haferflocken, Seefisch, Milch, Innereien und Fleisch, vor allem Rindfleisch. Zink aus tierischen Nahrungsmitteln wird vom Menschen besser verwertet als das aus pflanzlichen Nahrungsmitteln.

Unterschieden werden ein hereditärer und erworbener Zinkmangel. Ursache für den erworbenen Zinkmangel sind eine mangelhafte Resorption bei Malabsorptionssyndrom, z. B. bei chronischen Leber-, Pankreas- und Darmerkrankungen. Häufige Ursachen des Zinkmangels sind auch eine einseitige, $z$. B. vegetarische oder vegane Ernährung, weiterhin eine phytatreiche Ernährung, die überwiegend aus Vollkorn- und Sojaprodukten sowie zellulosereichen Lebensmitteln besteht, da diese die Zinkresorption im Ileum hemmen. Alkoholabusus, Laxantienabusus und eine parenterale Ernährung ohne Zink führen ebenfalls zu einem Zinkmangel. Eine verstärkte Ausscheidung von Zink wird hingegen beobachtet bei der Leberzirrhose und Hypalbuminämie, tubulären Nierenerkrankungen und Dialyse. Auch Krankheiten, die mit einem verstärkten Katabolismus einhergehen, wie Malignome, Chemotherapie, Spätstadien von AIDS, Traumen und Verbrennungen können zu einem drastischen Zinkmangel führen. Die Zinkausscheidung erfolgt zu 90\% über den Darm, weiterhin über die Niere, den Schweiß, das Nagel- und Haarwachstum und Sperma. In der Literatur wird auch darauf hingewiesen, dass auch häufige Ejakulationen ( $9 \mu \mathrm{mol}$ Zink pro Ejakulat) eine wichtige Quelle für einen Zinkverlust darstellen können. Schließlich können auch Medikamente, u. a. Kortikoide, Сyclosporin A, Diuretika, Eisen, Kontrazeptiva, ACE-Hemmer und Lipidsenker einen Zinkmangel begünstigen.

Beim hereditären Zinkmangelsyndrom handelt es sich um eine autosomal-rezessive Erkrankung. Infolge eines mutierten Transportproteins ist die Zinkresorption im Darm stark gestört.

Differenzialdiagnostisch abzugrenzen sind von der Zinkmangeldermatitis u. a. das seborrhoische Ekzem, die atopische Dermatitis, die Psoriasis vulgaris, das Exsikkationsekzematid, der Pemphigus foliaceus und erythematosus, das Erythema necroticans migrans und hereditäre Epidermolysen.

Grundsätzlich sollte bei therapieresistenten, akral betonten Hautveränderungen auch an eine Zinkmangeldermatitis ge- 
dacht werden und bei Bestätigung dieser Diagnose eine Ursachenklärung angeschlossen werden.

\section{Abstract}

\section{Zinc Deficiency Disorder}

$\nabla$

The presence of dermatitis in acral distribution accompanied by alopecia and diarrhea should raise the question of possible zinc deficiency. A zinc deficiency is accompanied by a low concentration of alcalic phosphatase. It is important to search for disorders which can be responsible for the zinc deficiency.

\section{Literatur}

1 Braun-Falco 0 et al. Dermatologie und Venerologie. 5. Auflage. Heidelberg: Springer Medizin Verlag, 2005: 1171 - 1173

2 Orfanos CE, Garbe C. Therapie der Hautkrankheiten. 2. Auflage. Heidelberg: Springer Verlag, 2001: 764- 765

3 Fitzpatrick TB et al. Dermatology in general medicine. 5. edition. New York: McGraw Hill, 1999: 1738 - 1743

4 Moschella $S$ et al. Dermatology. 3. edition. New York: McGraw Hill, 1992: $1617-1621$

5 Lausmann K. Langzeitversuch zur Knochenwirkung einer marginalen Zinkversorgung an adulten Ratten. Inaugural-Dissertation. Tierärztliche Fakultät der Ludwig-Maximilian-Universität. München: 2004 